\title{
Comparaison de systèmes d'analyse d'images cytologiques en dosimétrie biologique
}

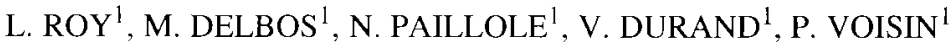

(Manuscrit reçu le 3 décembre 2002, accepté le 19 mars 2003)

RÉSUMÉ La technique de référence en dosimétrie biologique est basée sur le dénombrement des aberrations chromosomiques de type dicentrique induit par les rayonnements ionisants. Cet article présente divers systèmes d'analyse d'images utilisés en dosimétrie biologique pour aider la détection de ces aberrations. Les systèmes présentés sont le CYTOGEN de la société IMSTAR, le CYTOSCAN (APPLIED IMAGING) et le METAFER (METASYSTEM). Tous ne présentent pas les mêmes fonctionnalités et chacun peut être utilisé de façon plus ou moins automatique. Certaines fonctionnalités communes de ces systèmes sont comparées. L'aide apportée par les systèmes porte sur 3 points : (1) localisation automatique des métaphases sur les lames, dans ce cas on a un gain de temps d'un facteur 2 à 4 par rapport au comptage manuel ; (2) un outil d'aide au comptage qui apporte un confort de lecture et une meilleure fiabilité des résultats; (3) la détection automatique des dicentriques est particulièrement utile en cas de tri de population. En effet, dans ce cas il faut estimer très rapidement la dose reçue par un nombre important de personnes. Par contre, l'estimation de dose n'a pas besoin d'être aussi précise que dans le cas de l'expertise individuelle. Des erreurs dans la détection des dicentriques est alors tolérée et une détection automatique des dicentriques est envisageable. Le gain de temps est très appréciable puisqu'il est possible de compter 300 cellules en une demie-heure (METAFER) contre 25 avec la seule aide du chercheur de métaphases. Cependant la qualité de la détection doit encore être améliorée puisque $50 \%$ des dicentriques ne sont pas détectées. Le marquage des centromères par technique FISH devrait permettre d'améliorer la sensibilité de la technique. Les premiers résultats sont encourageant puisque $90 \%$ des centromères sont correctements détectés mais d'autres expériences doivent être réalisées pour évaluer le gain de temps.

ABSTRACT Comparison of cytological image analysis systems in biological dosimetry.

In biological dosimetry the reference technique is based on the scoring of dicentics and centric rings induced by ionizing radiation. This article tends to present some image analysis systems used in biological dosimetry to help aberration detection. The presented systems are: the CYTOGEN from IMSTAR society, the CYTOSCAN (APPLIED IMAGING) and the METAFER (METASYSTEM). All 3 systems do not present similar functionalities but offer different way of automation. Some functionalities of these systems are compared. The systems can assist operators on 3 major points: (1) an automatic metaphase finder, in this case, the time benefic ranges from 2 to 4 when compared to manual scoring; (2) a specific tool to help manual scoring of aberrations, this provide an easier scoring and a better stability of

\footnotetext{
1 IRSN. Département de protection de la santé de l'homme et de dosiméricic, Scction autonome de radiobiologie appliquée à la médecine, B.P. 17, 92262 Fontenay-aux-Roses Ccdex, France.
} 
the results; (3) the automatic detection of dicentrics, very useful in case of population triage. In this case, the dose has to be established very quickly, on a large population. However, the precision on the dose can be reduced compared to individual dose estimation. Some uncertainties on the detection of dicentric are acceptable and dicentric detection can be automated. Time benefic is then very important as 300 cells can be analyzed in half an hour (METAFER) against 25 when the metaphase finder is used alone. However, only $50 \%$ of the dicentrics are detected therefore it would be interesting to increase the efficiency of the detection. The use of the FISH technique could allow a better detection of dicentrics, the first results are promising ( $90 \%$ of centromeres correctly detected) but more experiments are required to evaluate the time benefit.

\section{Introduction}

La dosimétrie biologique consiste à mesurer, après exposition aux rayonnements ionisants, les variations radio-induites de certains paramètres biologiques et à permettre l'estimation d'une dose au travers d'une courbe dose-effet de référence (IAEA, 2001). Dans la cellule, la molécule d'ADN est une cible majeure des rayonnements ionisants. Ainsi, lorsqu'elle est lésée puis réparée, il peut y avoir formation d'aberrations chromosomiques. Les aberrations instables comme les dicentriques et les anneaux centriques sont utilisées en dosimétrie biologique comme indicateur de dose (Voisin et al., 2001).

Afin de pouvoir faire une estimation de dose en cas d'irradiation accidentelle, il est nécessaire de construire des courbes de référence dose/effet propres à chaque laboratoire pour différents types de rayonnement et différents débits de dose. Comme l'événement radio-induit recherché est un événement rare, un grand nombre de cellules doit être analysé, tant pour la réalisation de courbes dose/effet que pour une estimation de dose suite à un accident d'irradiation. Pour avoir des résultats exploitables statistiquement, il est nécessaire de rechercher les dicentriques dans au moins 500 cellules (Voisin et al., 2001). Des systèmes d'analyses d'images ont donc été développés en cytogénétique conventionnelle afin d'aider les cytogénéticiens dans cette tâche. Même si un nombre significatif de prototypes ont été mis au point dans différents laboratoires (Rutovitz, 1992), peu ont été commercialisés pour répondre spécifiquement aux besoins de la dosimétrie biologique. Il existe actuellement sur le marché le CytoVision ${ }^{\mathrm{TM}}$ (Applied Imaging) qui est une nouvelle version du CYTOSCAN auquel a été intégré le MAGISCAN décrit par Odawara et al. (1997). Ce système était disponible au laboratoire dans une version ancienne qui ne permettait pas l'analyse en fluorescence, et qui était utilisé pour la détection des métaphases depuis 1992. La société METASYSTEM commercialise aussi un système d'analyse d'images dédié à la cytogénétique (METAFER). Il est le seul à permettre une détection automatique des dicentriques, mais nous semblait peu évolutif. Nous avons donc cherché, en collaboration avec la société IMSTAR, à développer un système 
d'analyse d'images adapté à la dosimétrie biologique. Les étapes permettant le dénombrement des dicentriques à l'aide d'un système d'analyse d'image sont les suivantes : (1) un module de recherche de métaphases ; (2) un module d'acquisition d'images à fort grossissement pour servir de support au comptage semi-automatique des chromosomes et des aberrations (METAFER et CYTOGEN) ; (3) un module d'aide au comptage des dicentriques (CYTOGEN) ; (4) un module de détection automatique des dicentriques en fond clair et en fluorescence (METAFER et CYTOGEN).

Avant toute utilisation d'un nouveau système d'analyse d'images en dosimétrie biologique, il est nécessaire de le valider afin de s'assurer qu'il n'introduit pas de biais statistique. En effet, l'utilisation du système d'analyse d'images ne doit pas modifier la valeur de la dose obtenue par une observation manuelle. C'est pourquoi, dans un premier temps, le CYTOGEN est comparé avec le CYTOSCAN (Applied Imaging) développé par le groupe de Rutovitz (1992). Celui-ci est déjà utilisé dans plusieurs laboratoires, dont le nôtre, depuis quelques années, pour sa partie recherche automatique des métaphases en fond clair. Dans un deuxième temps, la partie recherche des métaphases en fluorescence du CYTOGEN est validée par rapport au même chercheur de métaphases mais en fond clair. Dans un troisième temps les performances de l'acquisition à fort grossissement sont présentées. La dernière partie porte sur la détection des dicentriques. Un outil de comptage semi-automatique ainsi que deux outils de détection automatique des dicentriques sont présentés, l'un en fluorescence (CYTOGEN) et l'autre en lumière transmise. En effet, le laboratoire dispose du système développé par METASYSTEM (METAFER, Allemagne), qui permet la recherche des métaphases et la détection automatique des dicentriques par la technique conventionnelle en coloration homogène en se basant sur l'analyse morphologique des chromosomes. Les deux approches seront donc présentées dans cet article.

\section{Matériel et méthodes}

\subsection{Matériel biologique}

\subsubsection{Culture cellulaire}

L'analyse des aberrations chromosomiques se fait sur des lymphocytes du sang périphérique mis en culture selon une technique décrite précédemment (Durand et al., 1995). En bref, ces cultures sont faites à partir de $0,5 \mathrm{ml}$ de sang total dans $5 \mathrm{ml}$ de milieu de culture complet (RPMI 1640, $10 \%$ de sérum de veau foetal, $1 \%$ de L-glutamine, $1 \%$ de pyruvate de sodium, $1 \%$ d'Hépès et de la PHA). Après 
46 heures de culture à $37^{\circ} \mathrm{C}$, les lymphocytes sont bloqués au stade de la métaphase par de la démécolcine $\left(0,5 \mu \mathrm{l} \mathrm{m}^{-1}\right)$ pendant 2 heures. Les étalements sur lames sont obtenus après un choc hypotonique $(0,075 \mathrm{M}$ de $\mathrm{KCl})$ et trois étapes de fixation méthanol/acide acétique $(3 / 1, v / v)$.

\subsubsection{Colorations}

Les étalements cellulaires sur lames sont colorés au Giemsa (technique F.P.G., Fluorescence Plus Giemsa) pour dénombrer les aberrations chromosomiques instables (dicentriques : IAEA, 2001).

Pour comparer directement les performances d'un même système en fond clair ou en fluorescence, un même stock de préparations microscopiques a été utilisé. Les lames ont été colorées au Giemsa, décolorées dans l'alcool et recolorées en DAPI (4'-6-diamine-2-phenylindole, $30 \mu \mathrm{l}$ sur la lame à une concentration de $0.02 \mu \mathrm{g} \mu \mathrm{l}^{-1}$ ). Ces cellules ont été étalées sur une petite surface de préparation afin de pouvoir plus facilement définir la zone de dénombrement des cellules.

\subsubsection{Hybridation in situ Fluorescente}

Les centromères sont marqués par une sonde couplée à la digoxigénine afin de détecter les dicentriques (spot fluorescent surnuméraire sur les chromosomes).

Le protocole a déjà été décrit par ailleurs (Roy et al., 1996). Brièvement, les lames sont d'abord prétraitées ( $\mathrm{RNase} \mathrm{A}$, pepsine et $\mathrm{MgCl}_{2}$ ). Les sondes sont déposées sur les lames et l'ensemble est dénaturé sur plaque chauffante à $72{ }^{\circ} \mathrm{C}$ et incubé toute la nuit à $37^{\circ} \mathrm{C}$. La sonde centromérique nécessite une détection par un anti-corps anti-digoxigénine couplé au FITC. Afin de pouvoir observer tous les chromosomes, les lames sont contre-colorées avec du DAPI.

\subsection{Matériel informatique}

\subsubsection{CYTOGEN}

Commercialisé par la société IMSTAR, ce système permet la recherche en lumière transmise et en fluorescence des métaphases, et l'observation des aberrations chromosomiques à fort grossissement.

Matériel : chaque poste est constitué d'un ordinateur de type PC, d'un microscope (SA, NIKON ou PROVIS, OLYMPUS) surmonté d'une caméra noir et blanc et d'une platine motorisée (PRIOR, Grande-Bretagne) en $x, y, z$ (pas en $x$, $y$ de $1 \mu \mathrm{m}$ et en $z$ de $2 \mu \mathrm{m}$ ). Quatre postes sont disponibles au laboratoire, chacun 
étant équipé soit d'une caméra COHU (high performance CCD caméra, non refroidie, $752 \times 582$ pixels) soit d'une HAMAMATSU (4742, refroidie par air à $0{ }^{\circ} \mathrm{C}, 1280 \times 1024$ pixels). Il n'a pas été mis en évidence d'influence de la caméra sur les résultats, si ce n'est une variation de la vitesse de fonctionnement, le nombre de pixels, donc le champs des deux types de caméra étant différent. La plupart des expérimentations présentées ici ont été réalisées sur les postes équipés d'une caméra HAMAMATSU.

Un objectif $\times 10$ (Fluor, NIKON) sert à la recherche des métaphases en fond clair et en fluorescence et un objectif $\times 60$ (Plan-Apo, NIKON) est utilisé pour l'analyse des aberrations à fort grossissement.

Fonctionnement : la recherche des métaphases nécessite les étapes suivantes :

- sur chaque lame, une zone de recherche des métaphases est définie ;

- l'utilisateur positionne au moins trois repères distants de mise au point. Le système va ensuite calculer le plan de netteté qui servira de référence pour la mise au point sur toute la zone de recherche ;

- le réglage des paramètres propres à la reconnaissance des métaphases :

- seuil manuel du niveau de gris à partir duquel l'objet sera analysé (valeur comprise entre 0 et 255 ),

- critère de taille des objets,

- critère de complexité de chaque groupement, basé sur l'indice de fractalité, les objets dont la forme sera trop simple ne seront pas sélectionnés,

- facteur de dilatation des petits objets pour les assembler en un groupement,

- autre critère de taille,

- critère de la forme de l'objet (rapport hauteur sur largeur, forme par rapport à un rectangle).

Le réglage de ces paramètres va permettre l'adaptation du système à chaque type de lame, indépendamment de la forme des métaphases, de leur superficie, de l'intensité de la coloration... L'utilisateur a la possibilité de tester la pertinence du réglage de ces paramètres.

La recherche en fluorescence fonctionne sur le même principe que la recherche en fond clair.

Analyse en fond clair : l'analyse des aberrations chromosomiques en fond clair nécessite l'acquisition des images à fort grossissement. Le système va utiliser la liste des positions provenant du chercheur de métaphases, pour placer chaque métaphase, une par une, sous l'objectif $\times 60$, faire un auto-focus, recentrer et acquérir l'image correspondante. Le système effectue une première détection des chromosomes et les marque comme étant «normaux ». L'utilisateur doit alors 
passer en revue chaque image et faire les corrections éventuelles correspondant aux aberrations présentes. À l'issue de cette opération, un tableau de résultats est édité indiquant le nombre de dicentriques, anneaux centriques, fragments dans les métaphases complètes (46 chromosomes).

Analyse en fluorescence : la roue porte filtre placée entre la lampe au mercure et l'échantillon à analyser, permet de sélectionner la longueur d'onde d'excitation spécifique de chaque fluorochrome (filtres simple bande, CHROMA). Comme nous fonctionnons avec des caméras noir et blanc, il est nécessaire de stocker autant d'images que de fluorochromes utilisés (ici 2). Elles sont ensuite superposées afin de reconstituer l'image en pseudo couleurs. Afin d'automatiser le changement des filtres la roue à filtre est pilotée par l'ordinateur. D'autre part, le bloc filtre triple-bande, placé entre l'échantillon et la caméra, a été modifié de façon à ne garder que le miroir dichrö̈que et le filtre d'arrêt, le filtre d'excitation ayant été retiré. Les caméras utilisées sont des caméras à accumulation ce qui permet d'intégrer le signal sur une période de temps suffisante pour pouvoir détecter des signaux de fluorescence très faibles.

Détection des dicentriques en fluorescence : le système de détection des dicentriques est basé sur la recherche automatique des métaphases, l'acquisition des images à fort grossissement, la détection des contours des chromosomes ainsi que la détection des centromères. La détection des contours chromosomes (rouge) et des centromères (bleu) n'a pas lieu sur les mêmes images. Les chromosomes sont détectés sur les images DAPI (Figs. 1A et 1C) tandis que les centromères sont observés sur les images FITC (Figs. 1B et 1D). Les contours sont alors superposés comme présenté en figure $1 \mathrm{E}$.

Des protocoles de détection ont été adaptés à notre type de marquage : ils comprennent des étapes de lavage d'images, de filtrage et de séparation d'objets.

\subsubsection{CYTOSCAN}

Il a été conçu par l'équipe de Rutovitz (Strark et al., 1989) dans les années 1980 et commercialisé par la société APPLIED IMAGING.

La version disponible au laboratoire est une ancienne version qui est basée sur le système d'exploitation OS 9. La conception de la recherche des métaphases est différente de celle du CYTOGEN puisqu'une barrette de diodes capture les images avec un microscope inversé (DIAPHOT, NIKON, France). Les groupes d'objets 
A

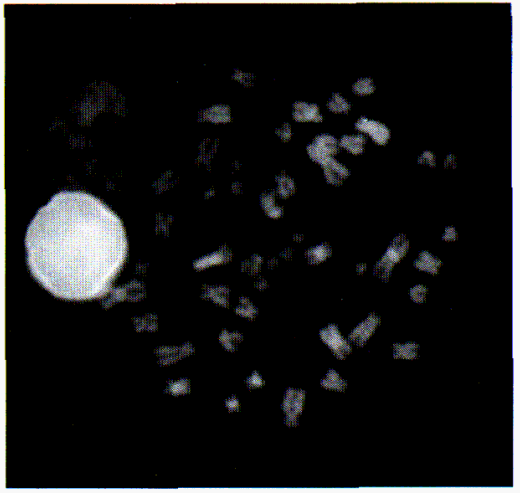

$\mathrm{C}$

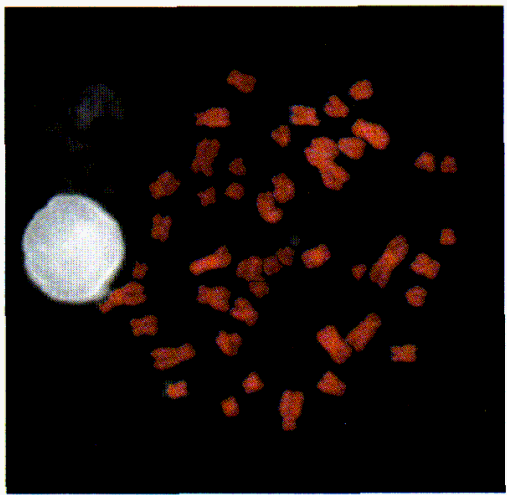

B

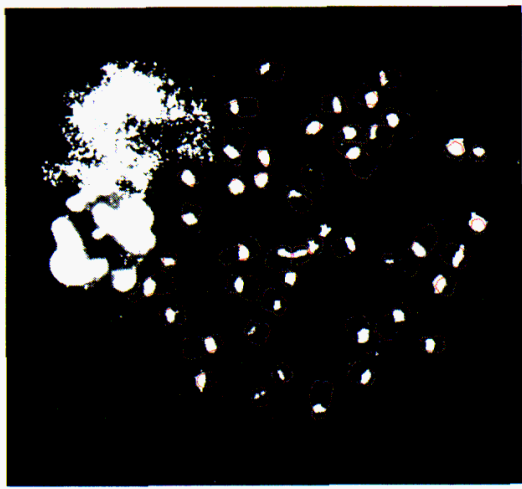

D

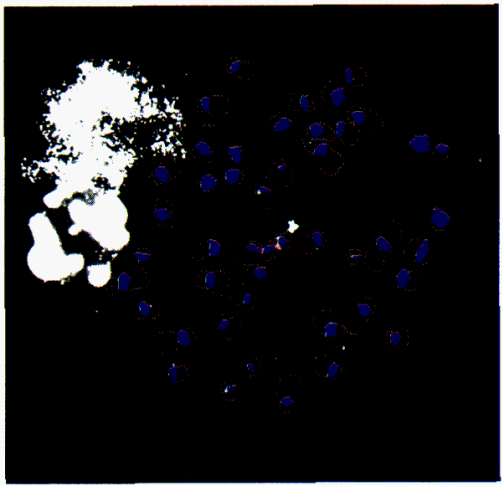

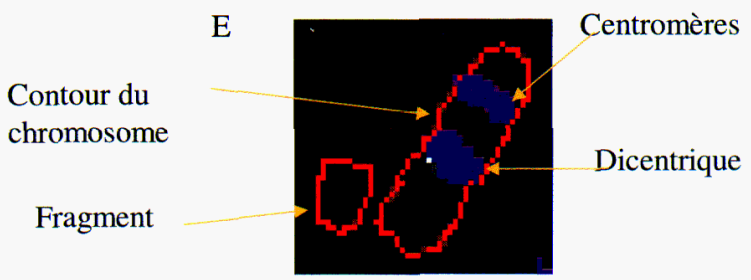

Figure 1 - Processus de détection des dicentriques par le système CYTOGEN. A : image des chromosomes (DAPI) ; B : image des centromères (FITC) ; $C$ : détection des contours des chromosomes (rouge) sur l'image DAPI ; $D$ : détection des contours des centromères (bleu); $E$ : détection des dicentriques basée sur la superposition des 2 contours.

Detection of dicentrics by the CYTOGEN system. A: visualization of the DAPI image (chromosomes); B: FITC image of centromeres; C: the chromosomes are detected on the DAPI image (red); D: automatic centromere detection on the FITC image; E: dicentric identification based on DAPI and FITC borders. 

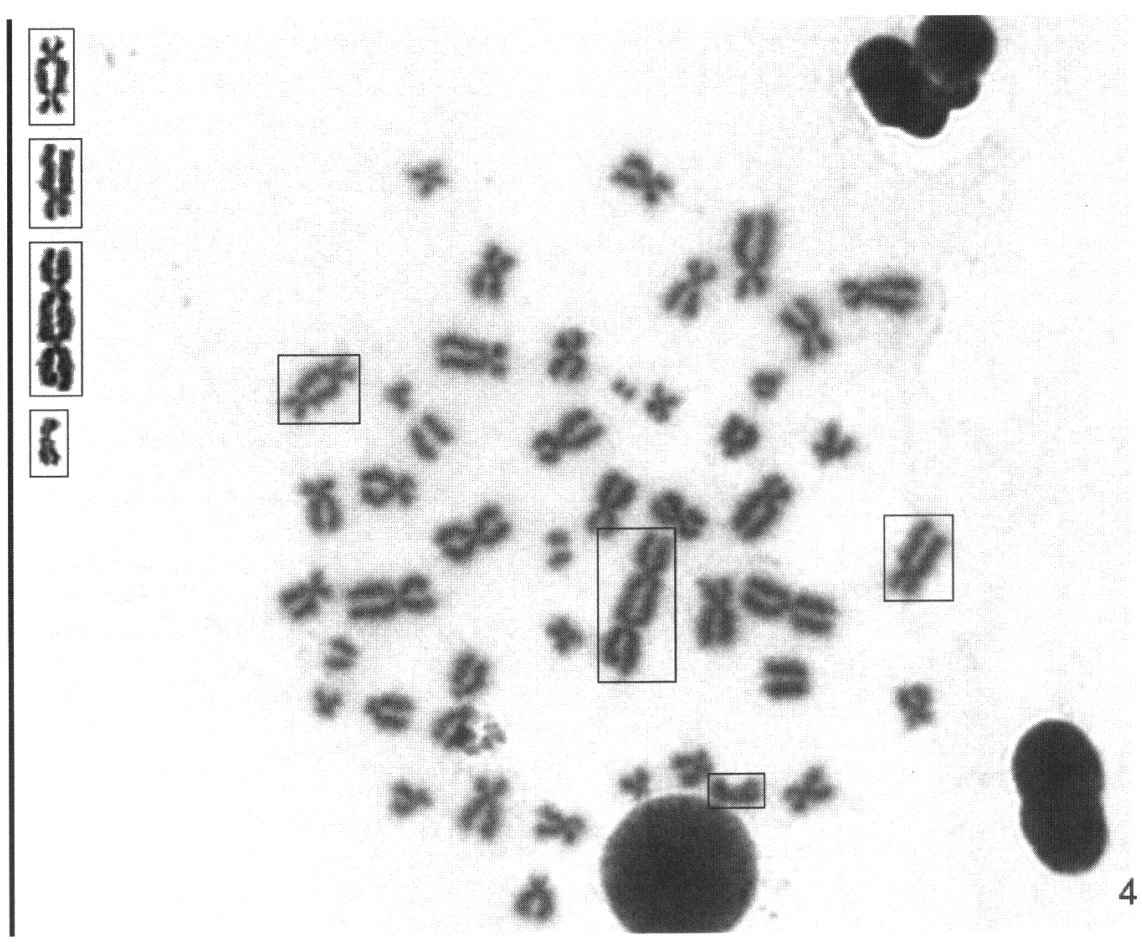

Figure 2-Image d'une métaphase acquise par le METAFER. Les objets encadrés correspondent aux chromosomes détectés comme dicentriques par le système. On remarque que deux de ces chromosomes ne sont pas des dicentriques.

Image of a metaphase stored by the METAFER. The outlines objects corresponds to presumed dicentrics. Among them, two are not real dicentrics.

sont repérés et analysés afin de décider s’ils forment ou non une métaphase à partir d'une succession de critères proches de ceux utilisés par le CYTOGEN.

Ce poste est utilisé dans le laboratoire comme chercheur de métaphases en fond clair. Il nous a servi comme référence pour valider les performances de la partie chercheur de métaphases du CYTOGEN.

\subsubsection{METAFER}

Commercialisé par la société METASYSTEM (Allemagne, Huber et al. 2001), ce système est conçu de la même façon que le CYTOGEN. Il permet d'abord une recherche automatique des métaphases en lumière transmise puis une acquisition à fort grossissement. Sur les images acquises, les chromosomes sont segmentés et 
les dicentriques détectés sur des critères de forme (profil en niveau de gris). Les candidats sont proposés à l'utilisateur qui décide en dernier ressort (Fig. 2). Puisque c'est un système qui doit être utilisé de façon automatique, nous avons souhaité évaluer sa qualité de détection. Pour ce faire, une courbe dose-effet a été réalisée à partir de quatre doses différentes. Pour chaque dose, neuf échantillons différents ont été comptés. Lors de l'analyse des dicentriques, le système effectue le contour des chromosomes, certains chromosomes mal définis sont alors éliminés. Pour qu'une cellule soit retenue, le nombre de chromosomes dans la cellule ne doit pas nécessairement être 46 comme pour le comptage manuel, toutes les cellules sont prises en compte quelque soit le nombre de chromosomes détectés. La fréquence des dicentriques est rapportée au nombre de chromosomes et non au nombre de cellules. Pour l'établissement de la courbe dose-effet un équivalent de 1500 cellules a été observé pour chaque point de dose (soit $1500 \times 46$ chromosomes).

\section{Résultats et discussion}

Notre objectif étant l'évaluation du CYTOGEN à des fins de dosimétrie biologique, deux points sont fondamentaux: la recherche des métaphases et l'analyse des aberrations. Les résultats sont donc séparés en deux parties, dans un premier temps, la partie recherche des métaphases du CYTOGEN a été testée par rapport au CYTOSCAN (Finnon et al., 1986) utilisé en routine dans le laboratoire depuis 1992. Dans un deuxième temps, la partie analyse des aberrations du CYTOGEN a été comparée à la détection automatique du METAFER.

\subsection{Chercheur de métaphases en fluorescence et en fond clair}

\subsubsection{Performance de vitesse des chercheurs}

Le réglage des paramètres avant une recherche de métaphases nécessite cinq fois plus de temps avec le CYTOGEN qu'avec les autres systèmes (METAFER et CYTOSCAN). En effet, avec le CYTOGEN, l'utilisateur a facilement accès aux critères de sélection des métaphases et cherche donc à les ajuster le mieux possible à la préparation microscopique. Ceci prend plus de temps qu'avec les deux autres systèmes pour lesquels les réglages sont plus difficilement accessibles. De plus, dans le cas du CYTOSCAN et du METAFER aucun test ne peut être fait pour connaître la pertinence de ces réglages, l'utilisateur a tendance à ne pas adapter les paramètres de recherche en fonction de la qualité des lames, ce qui justifie la durée plus courte des réglages préliminaires. Par contre, le réglage des paramètres sur le CYTOGEN requière du personnel expérimentés. L'influence possible du réglage des paramètres sur la qualité de la recherche sera discuté ultérieurement. 


\section{TABLEAU I}

Comparaison des performances obtenues par le CYTOSCAN et le CYTOGEN en fond clair : nombre d'objets détectés comme étant des métaphases par le chercheur, pourcentage de faux positifs parmi ces objets et pourcentage de faux négatifs. Les valeurs entre parenthèse représentent les valeurs minimales et maximales obtenues.

Comparison of the performances of the CYTOGEN and the CYTOSCAN in bright field: number of metaphases found, percentage of false positive and percentage of false negative. Values in brackets are minimum and maximum values of both percentages.

\begin{tabular}{lcc}
\hline & CYTOGEN & CYTOSCAN \\
\hline Nombre moyen d'objets & 653 & 147 \\
Pourcentage de faux positifs & $8 \%($ entre 1 et $55,2 \%)$ & $2 \%($ entre 0 et $19 \%)$ \\
Pourcentage de faux négatifs & $38 \%($ entre 20 et $50 \%)$ & $84 \%($ entre 43 et $94 \%)$ \\
\hline
\end{tabular}

À cette phase qui nécessite l'intervention humaine, succède la phase de recherche automatique des métaphases. La durée de la recherche varie en fonction de la conception du système et des performances de la platine motorisée. Le temps de recherche des métaphases avec le CYTOSCAN est de $1 \mathrm{~min} \mathrm{~cm}^{-2}$. Ce système a longtemps été le plus rapide du marché du fait de son processeur en tranche spécialement conçu pour analyser les images en parallèle (Strark et al., 1989). Actuellement l'amélioration de la conception des platines fonctionnant avec un PC standard, permet d'atteindre une vitesse du même ordre de grandeur (1,7 $\mathrm{min} \mathrm{cm}^{-2}$ CYTOGEN et METAFER) alors que précédemment, le rapport était de 10 .

\subsubsection{Performance de détection des métaphases en fond clair}

Les performances du CYTOGEN ont été comparées à celles du CYTOSCAN en ce qui concerne le taux de faux négatifs (pourcentage de métaphases réellement présentes sur la lame mais non détectées par le système) et le taux de faux positifs (pourcentage d'objets qui ne sont pas des métaphases parmi les objets que le système a trouvé). La zone de recherche est identique pour les dix lames et pour les deux systèmes. Les résultats obtenus sont résumés dans le tableau I. La première ligne correspond à la somme des objets détectés sur les 10 lames. Sur la deuxième ligne, les positions trouvées par le système ont été vérifiées par l'utilisateur ce qui permet de calculer le pourcentage moyen de faux positifs observé sur les lames avec entre parenthèse la valeur maximale et minimale de ce taux. Dans un deuxième temps, la lame a été parcourue manuellement et les métaphases ont été dénombrées. On en déduit alors le pourcentage de faux négatifs (voir Sect. 2). On constate que le CYTOGEN trouve 4,5 fois plus d'objets que le CYTOSCAN et bien que le taux de faux positifs soit légèrement supérieur ( $8 \%$ contre $2 \%$ ), le CYTOGEN trouve au total 4 fois plus de métaphases que le CYTOSCAN. Ceci montre l'importance de pouvoir adapter facilement les 
paramètres de la recherche suivant la qualité de la lame, ce qui est le cas avec le CYTOGEN, et justifie le temps plus long dédié aux réglages de l'appareil. La majorité des faux positifs provient de taches de colorant pouvant répondre aux mêmes critères que les métaphases. Il serait possible de diminuer encore le taux de faux positifs trouvés par le CYTOGEN en faisant des réglages plus fins des paramètres en fonction des lames. Néanmoins il faut trouver un compromis entre le pourcentage de faux positifs acceptables et le temps passé pour ajuster les paramètres pour la recherche.

La fluctuation du pourcentage de faux positifs suivant les lames est plus important pour le CYTOGEN (de 1 à 55,2 \%) que pour le CYTOSCAN (de 0 à $19 \%$ ). Ceci est dû au fait que le CYTOSCAN selectionne uniquement les belles métaphases quelque soit la lame avec peu d'erreurs. Ainsi le pourcentage de métaphases non détectées est très élevé. Par contre, le CYTOGEN sélectionne plus d'objets avec comme conséquence de faire plus d'erreurs et d'être très influencé par les réglages et la qualité de l'étalement.

On peut constater qu'il reste toujours des métaphases qui ne sont pas détectées. La question est alors de savoir si ce sont à la fois des métaphases avec aberrations et sans ou si un biais statistique est introduit du fait de l'utilisation du chercheur de métaphases qui sélectionnerait spécifiquement un type de cellules (normal ou anormal).

\subsubsection{Performances de détection des métaphases en fluorescence}

Dans un deuxième temps ont été validées les performances du CYTOGEN en fluorescence. Ne disposant pas, au laboratoire, d'un autre chercheur de métaphases en fluorescence, le CYTOGEN a été comparé à lui-même. Le procédé de la recherche est le même dans les deux cas, sauf en ce qui concerne les valeurs du seuil des niveaux de gris, puisqu'en fond clair, l'image est noire sur un fond blanc, alors qu'en fluorescence, les objets sont clairs sur un fond noir.

Le tableau II présente les résultats obtenus à partir de cinq lames qui ont été colorées successivement au Giemsa puis au DAPI. La durée de la recherche varie

\section{TABLEAU II}

Comparaison du pourcentage de faux positifs et de faux négatifs sur le CYTOGEN en fond clair et en fluorescence.

Comparison of the number of false positive, false negative of the CYTOGEN system in bright field and in fluorescence.

\begin{tabular}{|lcc|}
\hline & CYTOGEN en fond clair & CYTOGEN en fluorescence \\
\hline Pourcentage de faux positifs & $8 \%$ & $14 \%$ \\
\hline Pourcentage de faux négatifs & $38 \%$ & $28 \%$ \\
\hline
\end{tabular}


suivant le temps d'accumulation de la caméra, qui peu dépendre de la concentration en DAPI. Nous avons réglé la notre pour que l'exposition de la caméra pour la recherche des métaphases en fluorescence soit identique à celle en fond clair. La vitesse de recherche des métaphases est alors la même soit $1,7 \mathrm{~min}^{-2}$. Cette vitesse a pu être obtenue avec les platines de dernière génération. Elle est plus élevée que celle du chercheur de métaphases en fluorescence mis au point par Piper et al. (1994) qui est de $10 \mathrm{~min}^{-2}$.

On constate une augmentation du pourcentage de faux positifs en fluorescence par rapport au fond clair. Ceci pourrait provenir d'une diminution du niveau de contraste en fluorescence comparativement au fond clair. De plus, les réglages sont plus difficiles en fluorescence, à cause de la courte durée de vie d'émission de la fluorescence. À l'inverse, le pourcentage de faux négatifs (métaphases présentes sur la lame et que le système n'a pas trouvées) est inférieur en fluorescence qu'en fond clair. Ceci pourrait s'expliquer par des paramètres de recherche plus adaptés aux caractéristiques des métaphases en fluorescence.

Finalement, nous avons comparé la fréquence des dicentriques dans les métaphases localisées automatiquement et dans celles localisées manuellement. La fréquence des dicentriques n'est pas différente dans les deux cas. De plus, avec le chercheur de métaphases, on observe un gain de temps d'un facteur 2 à 4 par rapport à un comptage entièrement manuel. Les performances obtenues avec le chercheur de métaphases du CYTOGEN montrent que ce système peut être utilisé indifféremment en fond clair et en fluorescence.

\subsection{Acquisition}

Une fois que la liste des coordonnées des métaphases est obtenue par le chercheur de métaphases, celle-ci est relue en vue de l'acquisition des images à fort grossissement. Durant cette phase, le système ne nécessite aucune intervention de la part de l'utilisateur : l'auto-focus de chaque position, le centrage et l'acquisition des images sont faits automatiquement. Les réglages préliminaires à l'acquisition requièrent 5 minutes pour chaque liste de positions, ce temps comprend le chargement de la liste, le réglage des paramètres de la caméra, le positionnement de repères à fort grossissement. La durée de l'acquisition varie en fonction du nombre de métaphases à acquérir. Elle est en moyenne de 6 secondes par métaphase, soit une 10 minutes pour 100 images en lumière transmise. En lumière fluorescente, nous avons obtenus des valeurs similaires pour une durée d'exposition courte en DAPI et un bon marquage des chromosomes (FITC), par contre ces performances peuvent varier en fonction de l'intensité des fluorochromes. 
En fluorescence, il est nécessaire d'acquérir autant d'images que de fluorochromes présents sur la préparation. En cé qui concerne la détection des dicentriques un seul fluorochrome est utilisé en plus du DAPI, le FITC qui marque les centromères en vert, ce qui nécessite deux images par position.

Sur la liste des positions, $12 \%$ des métaphases ne sont plus exploitables à cause du processus d'acquisition avec le CYTOGEN. Ces métaphases sont rendues inexploitables car floues ou non centrées. Néanmoins, l'acquisition permet d'analyser des métaphases dont le signal fluorescent est faible et difficilement visible directement au microscope.

\subsection{Analyse des dicentriques}

\subsubsection{Aide au comptage}

Cet outil n'est disponible que sur le CYTOGEN, il permet un comptage automatique des chromosomes sur chaque métaphase colorée. Ces chromosomes sont classés, par défaut, comme normaux. L'utilisateur fait défiler les métaphases afin d'ajouter les chromosomes qui n'auraient pas été détectés ou de reclasser comme aberrants les dicentriques, les fragments et les anneaux centriques. Une vérification visuelle des aberrations est possible au microscope, si nécessaire. La durée pour corriger chaque métaphase va dépendre de la qualité de la métaphase et du nombre d'aberrations présentes. Le temps varie de 10 secondes pour une métaphase sans aberration où les chromosomes ont été bien détectés, jusqu'à 40 secondes s'il y a beaucoup de corrections à effectuer. Le CYTOGEN permet l'édition des résultats sous diverses formes, soit par métaphase analysée ou uniquement par métaphase complète (46 chromosomes). Ceci est d'autant plus convivial que seules les métaphases complètes doivent être prises en considération pour l'estimation de la dose.

En définitive, une fois les métaphases localisées automatiquement, l'utilisation de l'outil d'aide au comptage du CYTOGEN n'apporte qu'un gain faible par rapport au comptage manuel, c'est-à-dire d'un facteur 2 à 4 par rapport au comptage entièrement manuel (recherche manuelle). Par contre, le comptage des chromosomes à l'écran est moins contraignant qu'au microscope, l'opérateur est alors plus efficace. Cet outil est particulièrement utile pour l'analyse des fortes doses où il faut compter un grand nombre de dicentriques ce qui rend le comptage entièrement manuel difficile. Le fait d'utiliser un outil d'aide au comptage contribue à l'obtention de résultats plus fiables. Cette détection semi automatique des dicentriques est d'ailleurs l'une des originalités du système. 


\subsubsection{Détection automatique des dicentriques}

En cas d'accident de grande envergure, il faut identifier les personnes les plus atteintes afin d'effectuer un suivi médical adapté. La dosimétrie biologique est un des outils qui permet l'identification des ces personnes. Il faut alors analyser un grand nombre d'échantillons sanguins en un temps minimum, une précision de \pm 1 Gy étant suffisante. Pour cela seules 50 cellules sont analysées au lieu de 500 en situation d'expertise. Le laboratoire est ainsi capable de traiter 100 échantillons en 7 jour (Voisin et al., 2001). Mais ne serait-il pas possible grâce à l'automatisation d'être plus efficace (Bayley et al., 1991)?

Pour le tri d'urgence d'un nombre important de personnes, nous avons envisagé une détection automatique des dicentriques.

\section{Par coloration homogène}

Dans ce cas, la détection des dicentriques est basée sur l'analyse du profil des niveaux de gris des chromosomes afin de localiser la ou les constriction(s) présente(s) au niveau du centromère. Les premiers résultats obtenus avec le CYTOGEN ne sont pas reproductibles et le taux de faux positifs reste trop élevé.

Seul le METAFER propose une détection automatique des dicentriques par un marquage en coloration homogène (Fig. 2). Dans ce cas, la démarche consiste à faire une recherche automatique des métaphases, suivie d'une acquisition des images à fort grossissement. Le système analyse ensuite les profils des chromosomes et propose une galerie de dicentriques candidats qui sont vérifiés par l'utilisateur. Le résultat obtenu est un nombre de dicentriques par chromosome et non par cellule. Afin de comparer les résultats ainsi obtenus avec nos courbes de références établies manuellement, les chromosomes ont été regroupés par 46 afin d'avoir un taux de dicentriques par cellule équivalente. Près de 1500 cellules équivalentes ont été dénombrées pour chaque point. Une courbe dose-effet a été obtenue à partir de l'irradiation d'échantillons sanguins in vitro par une source de césium-137 à un débit de dose de $0,1 \mathrm{~Gy} \mathrm{~min}^{-1}$ (Fig. 3). Sur la figure, deux courbes ont été tracées, celle réalisée après détection automatique des dicentriques par le METAFER et celle réalisée manuellement dans les mêmes conditions d'irradiation. On remarque que la pente de la courbe réalisée avec l'aide du METAFER est nettement inférieure à la courbe équivalente réalisée manuellement. En effet, un certain nombre de dicentriques ne sont pas pris en compte du fait de leur morphologie. Mais puisque la courbe obtenue automatiquement reste linéaire quadratique, on peut en déduire que la perte d'information est répartie au hasard sur l'ensemble des points. L'utilisation de cette courbe en expertise est examinée actuellement au laboratoire. 


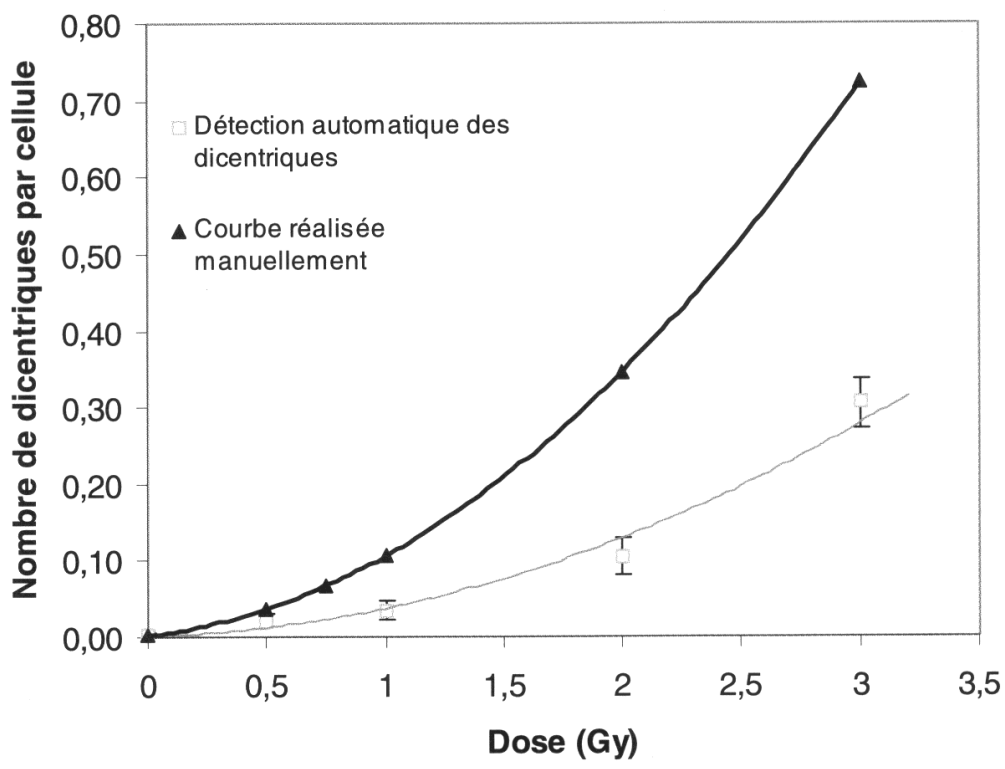

Figure 3 - Comparaison de la courbe de référence du laboratoire avec la courbe établie avec le METAFER en utilisant la détection automatique des dicentriques. Dans ce cas, le nombre de cellules est calculé en groupant les chromosomes en groupe de 46. Les barres d'erreur correspondent à l'intervalle de confiance à $95 \%$ de la mesure.

Comparison of the dose effect relationship established manually and by detecting dicentrics automatically with the METAFER system. In this case, the number of chromosome detected is divided by 46 in order to simulate cells. Error bars represent $95 \%$ confidant interval.

En terme de temps, il faut environ 0,5 heure pour vérifier 300 cellules avec le METAFER, ce qui est très performant par rapport au comptage manuel. En effet, durant la même durée il n'est possible de compter que 25 cellules avec l'aide d'un chercheur automatique de métaphases.

\section{Par marquage centromérique}

Le marquage centromérique par FISH peut aussi être utilisé pour automatiser la détection des dicentriques. Dans ce cas, les centromères sont marqués par une sonde fluorescente et un dicentrique est repéré comme un chromosome ayant deux spots fluorescents (Fig. 1).

Les premiers résultats obtenus avec le CYTOGEN montrent que $95 \%$ de l'ensemble des centromères est correctement détecté. Par contre, la détection des chromosomes est plus délicate car dépendante de la qualité de l'étalement et des 
TABLEAU III

Description des avantages et inconvénients des différentes façons d'utiliser les systèmes d'analyse d'images en fonction des niveaux d'automatisation choisis.

Description of advantages or inconvenient of the different way to use the system according to the level of automation.

\begin{tabular}{|c|c|c|c|c|c|}
\hline \multirow{6}{*}{$\begin{array}{l}\text { Processus } \\
\text { d'observation } \\
\text { des } \\
\text { dicentriques }\end{array}$} & \multicolumn{5}{|c|}{1 - Recherche des métaphases } \\
\hline & Manuelle & Automatique & Automatique & Automatique & Automatique \\
\hline & \multicolumn{5}{|c|}{2 - Acquisition } \\
\hline & - & - & Automatique & Automatique & Automatique \\
\hline & \multicolumn{5}{|c|}{3 - Dénombrement des dicentriques } \\
\hline & Manuel & Manuel & Aide au comptage & $\begin{array}{l}\text { Automatique } \\
\text { (fluorescence) }\end{array}$ & $\begin{array}{l}\text { Automatique } \\
\text { (coloration } \\
\text { homogène) }\end{array}$ \\
\hline \multirow[t]{2}{*}{ Avantages } & \multirow{2}{*}{$\begin{array}{l}\text { - Observation } \\
\text { de référence. }\end{array}$} & \multirow{2}{*}{$\begin{array}{l}\text { - Gain de } \\
\text { temps d'un } \\
\text { facteur } 2 \text { à } 4 .\end{array}$} & \multirow{2}{*}{$\begin{array}{l}\text { - Observation } \\
\text { plus fiable surtout } \\
\text { aux fortes doses. } \\
\text { - Permet le } \\
\text { stockage des } \\
\text { images à long } \\
\text { terme. }\end{array}$} & \multicolumn{2}{|l|}{ - Beaucoup plus rapide. } \\
\hline & & & & $\begin{array}{l}\text { - Détection possible } \\
\text { des dicentriques avec } \\
\text { un des centromères } \\
\text { au bout. } \\
\text { - Performances } \\
\text { de la détection } \\
\text { améliorables. }\end{array}$ & $\begin{array}{l}\text { - Outil de détection } \\
\text { automatique et de } \\
\text { vérification très } \\
\text { rapide. } \\
\text { - Coloration } \\
\text { conventionnelle à } \\
\text { valeur médico-légale. }\end{array}$ \\
\hline \multirow[t]{2}{*}{ Inconvénients } & \multirow[t]{2}{*}{ - Long } & \multirow[t]{2}{*}{ - Fatiguant } & & \multicolumn{2}{|c|}{$\begin{array}{l}\text { - Détection de seulement } 50 \% \\
\text { des dicentriques } \\
\text { - La fréquence des dicentriques par rapport } \\
\text { à un nombre de chromosomes et non par } \\
\text { cellule. }\end{array}$} \\
\hline & & & & $\begin{array}{l}\text { - Contour des } \\
\text { chromosomes moins } \\
\text { précis qu'en lumière } \\
\text { transmise. }\end{array}$ & $\begin{array}{l}\text { - Certaines formes de } \\
\text { dicentriques ne sont } \\
\text { pas reconnues. }\end{array}$ \\
\hline
\end{tabular}

chevauchements. Pour corriger les erreurs de détection sur les contours des chromosomes, en moyenne :

- 0 à 5 objets sont à enlever par cellule (moyenne, 0,7 ) ;

- 0 à 12 objets sont à séparer par cellule (moyenne, 5) ;

- 0 à 3 objets sont à dessiner par cellule (moyenne, 0,3 ).

À l'inverse, on peut préférer ignorer les amas de chromosomes et considérer la fréquence des dicentriques non par cellule mais par chromosome comme dans le cas du METAFER.

Des études préliminaires montrent que plus de $50 \%$ des dicentriques sont correctement détectés. Ces études se poursuivent afin d'améliorer ces performances notamment en associant au marquage centromérique un marquage télomérique qui permettrait de localiser le début et la fin de chaque chromosome. 


\section{Conclusion}

Plusieurs outils adaptés à la dosimetrie biologique ont été présentés dans cet article.

Le tableau III reprend les différentes façons d'utiliser les systèmes d'analyse d'images pour un niveau d'automatisation croissant. Ainsi, le chercheur automatique des métaphases est l'outil de base indispensable pour améliorer la vitesse de comptage. Il permet un gain de temps d'un facteur 2 à 4 par rapport au comptage manuel, celui-ci étant utilisé comme référence pour valider les systèmes et s'assurer qu'aucune dérive n'existe. L'outil d'aide au comptage permet d'être plus fiable surtout aux fortes doses d'irradiation mais permet aussi le stockage des images en vue d'un archivage nécessaire dans une démarche d'assurance qualité. Deux systèmes de détection automatique des dicentriques sont à l'étude au laboratoire, le METAFER permet une détection des dicentriques en coloration homogène. Il est très rapide mais le taux de dicentriques non détectés reste trop important. Par contre, le CYTOGEN, plus lent, devrait permettre une meilleure efficacité dans la détection des dicentriques. La prochaine étape reste donc la réalisation d'une courbe dose-effet pour ce système.

\section{RÉFÉRENCES}

Baylay R., Carothers A., Chen X., Farrow S., Gordon J., Ji L., Piper J., Rutoviyz D., Stark M., Wald N. (1991) Radation dosimetry by automatic image analysis of dicentric chromosomes, Mutat. Res. 253, 223-235.

Durand V., Chambrette V., Le Roy A., Paillole N., Sorokine-Durm I., Voisin P. (1995) La dosimétrie biologique en cas d'accident d'irradiation, Radioprotection 30, 305-310.

Finnon P., Lloyd D.C., Edwards A.A. (1986) An assessment of the melaphase finding capacity of the Cyloscan 110, Mutat. Res. 164, 10l-108.

Huber R., Kulka U., Lorch T., Braselmann H., Engert D., Figel M., Bauchinger M. (2001) Technical report: application of the Metafer 2 fluorescence scanning system for the analysis of radiationinduced chromosome abcrations measured by FISH-chromosome painting, Mutat. Res. 492 , $51-57$.

IAEA (2001) International Atomic Energy Agency Technical Report series $n^{\circ}$ 405, Cytogenetic Analysis for Radiation Dose Assessment, IAEA, Vienna.

Odawara K., Yamamoto K., Kato H., Hara M., Kogiso S., Kishida F., Yoshitake A., Nakatsuka I. (1997) A new semi-automated chromosome analysis system for in vitro chromosomal abcrration tests, Mutat. Res. 389, 207-212.

Piper J., Poggensee M., Hill W., Jensen R., Ji L., Poole I., Stark M., Sudar D. (1994) Automatic fluorescencc melaphase finder speeds translocation scoring in FISH painted chromosomes, Cytometry 16, 7-16.

Roy L., Sorokine-Durm I., Voisin P. (1996) Comparison belween fluorescence in situ hybridization and conventional cylogenetics for dicentric scoring: a first-step validation for the use of FISH in biological dosimetry, Int. J. Radiat. Biol. 70, 665-669. 


\section{ROY et al.}

Rutovitz D.J. (1992) Reflections on the past, present and future of automated aberration scoring systems for radiation dosimetry, J. Radiat. Res. 33, 1-30.

Strark M., Farrow S., McKie M., Rutovitz D. (1989) Automation of Cytogenetics (C. Lundsteen, J. Piper, Eds), pp. 31-43, Berlin.

Voisin P., Benderitter M., Claraz M., Chambrette V., Sorokine-Durm I., Delbos M., Durand V., Leroy A., Paillole N. (2001) The cytogenetic dosimetry of recent accidental overexposure, Cell. Mol. Biol. 3, 557-564. 